7 Storla DG, Yimer S, Bjune GA. A systematic review of delay in the diagnosis and treatment of tuberculosis. BMC Public Health 2008; 8: 15.

8 Borgdorff MW, van der Werf MJ, de Haas PEW, et al. Tuberculosis elimination in the Netherlands. Emerg Infect Dis 2005; 11: 597-602.

9 Brinkmann B, Du Chesne A, Vennemann B. A survey of autopsy rates in Germany. Dtsch Med Wochenschr 2002; 127: 791-795.
10 Lefebvre N, Falzon D. Risk factors for death among tuberculosis cases: analysis of European surveillance data. Eur Respir J 2008; 31: $1256-1260$.

\title{
Rituximab in bronchiolitis obliterans after haematopoietic stem cell transplantation
}

\section{To the Editors:}

Bronchiolitis obliterans (BO) following allogeneic haematopoietic stem cell transplantation (HSCT) is recognised as a pulmonary manifestation of chronic graft-versus-host disease (GVHD) [1, 2]. As lung biopsy is rarely performed in these patients, the diagnosis of BO syndrome (BOS) usually relies on pulmonary function testing (PFT) and computed tomography (CT) lung scans [1, 2]. Very little retrospective data exist regarding the treatment of BOS, which currently consists of increasing a patient's baseline dose of systemic immunosuppressive therapy; however, this treatment strategy has been shown to have poor efficacy [2,3]. The efficacy of new drugs for the treatment of chronic GVHD, including the anti-CD20 monoclonal antibody rituximab, must be specifically evaluated for BOS. Here, we report three cases in which patients received rituximab for $\mathrm{BO}$ after allogeneic HSCT.

Patient 1 was a 15-yr-old male who received bone marrow from a sibling human leukocyte antigen (HLA)-matched donor for acute myeloid leukaemia after a myeloablative conditioning regimen that included 12 Gy fractionated total body irradiation (FTBI) and cyclophosphamide. 5 months after transplantation, he was diagnosed with chronic liver GVHD that led to the initiation of prednisone at a dosage of $1 \mathrm{mg} \cdot \mathrm{kg}^{-1} \cdot \mathrm{day}^{-1}$ as well as tacrolimus. 8 months after transplantation, as his liver GVHD was coming under control, he presented with dyspnoea, dry cough and chest pain. At this time, he was receiving tacrolimus and prednisone at a dosage of $20 \mathrm{mg} \cdot \mathrm{day}^{-1}$. PFT revealed a severe obstructive pattern based on a forced expiratory volume in $1 \mathrm{~s}$ (FEV1) of $1.13 \mathrm{~L}$ (33\% predicted), a forced vital capacity (FVC) of $2.79 \mathrm{~L}$ (65\% pred) and FEV1/FVC ratio of $41 \%$. A lung CT scan demonstrated the presence of air trapping and a pneumomediastinum. A pulmonary infection was ruled out, and the diagnosis of post-allogeneic HSCT BOS was made. The patient presented with no other signs of chronic GVHD at this time. The prednisone dosage was increased to $2 \mathrm{mg} \cdot \mathrm{kg}^{-1} \cdot \mathrm{day}^{-1}$, tacrolimus treatment was continued, and mycophenolate mofetil, inhaled steroids, bronchodilators and low-dose macrolides were added to the treatment regimen. Despite this treatment and although the pneumomediastinum resolved, PFT revealed consistent deterioration over the following months. $4 \mathrm{yrs}$ after transplantation, the patient was still receiving $20 \mathrm{mg} \cdot$ day $^{-1}$ prednisone, inhaled steroids, bronchodilators and low-dose macrolides, and developed a left spontaneous pneumothorax that necessitated pleurectomy. A surgical lung biopsy was simultaneously performed. A histological examination confirmed the diagnosis of $\mathrm{BO}$ : several bronchioles were narrowed or completely obliterated by fibrosis, while others had walls containing lymphocytes, primarily B-cells (fig. 1). In response to these findings, the patient received two infusions
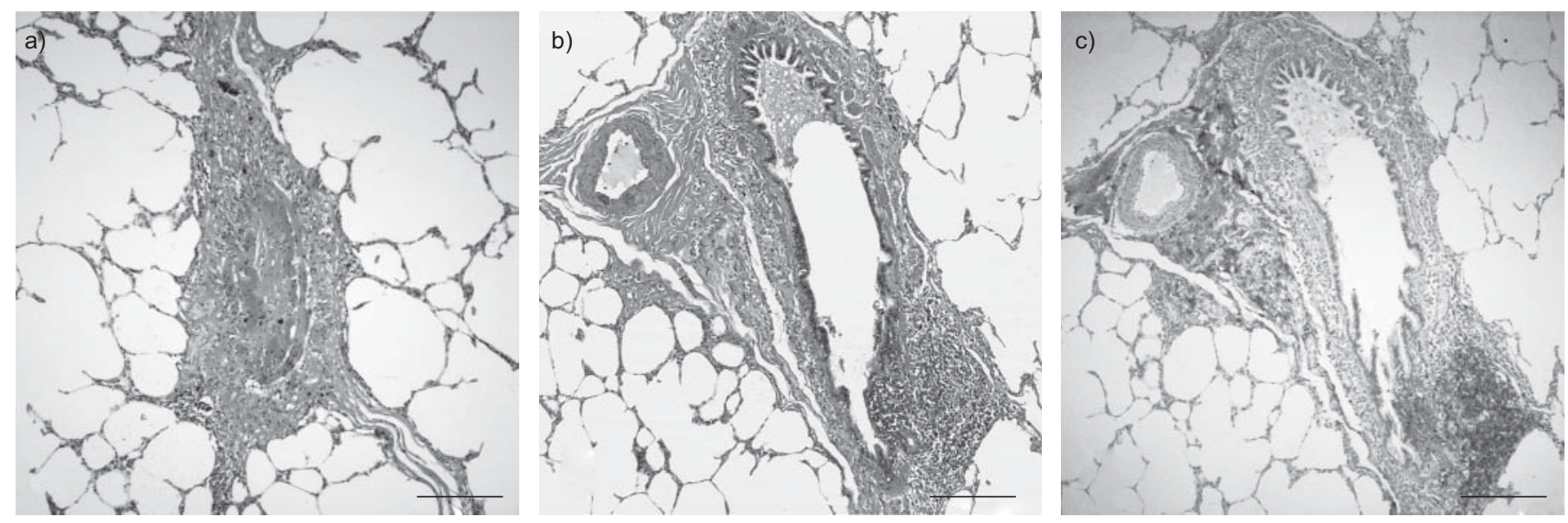

FIGURE 1. a) The lumen of this bronchiole is completely obliterated by dense fibrosis (haematoxylin and eosin staining (HES)). b) A nodular lymphoid infiltrate is found in the wall of this bronchiole (HES). c) The lymphocytes of the infiltrate are predominantly B-cells (immunohistochemistry with anti-CD20 antibody). Scale bars $=200 \mu \mathrm{m}$. 
of rituximab (1,000 mg each) on days 1 and 15. At this time, PFT revealed an FEV1 of $0.72 \mathrm{~L}$ ( $20 \%$ pred) and an FVC of $2.48 \mathrm{~L}(53 \%$ pred). 4 months after administration of rituximab, the patient's dyspnoea had not improved, and his PFT results were unchanged (fig. 2). 6 months later, hypercapnia had developed and bilateral pneumothoraces were found. The patient is currently awaiting lung transplantation.

Patient 2 was a 28-yr-old female who underwent a transplantation of peripheral blood stem cells (PBSCs) from an unrelated HLA-matched donor for acute lymphoid leukaemia after a myeloablative conditioning regimen that included FTBI and cyclophosphamide. The early course after transplantation was complicated by steroid-refractory skin GVHD, which was controlled with an anti-interleukin-2 receptor monoclonal antibody, high-dose methylprednisolone and tacrolimus. 5 months after the transplantation and 1 month after steroid treatment ended, the patient was still receiving tacrolimus and experienced a febrile pneumonia that was resolved with empirical broadspectrum antibiotics. 1 month later, the patient developed a dry cough, dyspnoea and wheezing. PFT revealed a severe obstructive pattern: FVC $1.34 \mathrm{~L}$ (37\% pred), FEV1 $0.84 \mathrm{~L}$ (27\% pred), FEV1/FVC 63\% and residual volume (RV) $2.84 \mathrm{~L}$ (203\% pred). The lung CT scan demonstrated the presence of air trapping and a pulmonary infection was ruled out. A diagnosis of BOS was made, as no other signs of GVHD were observed. The patient then received three pulses of methylprednisolone (500 mg each) followed by $1 \mathrm{mg} \cdot \mathrm{kg}^{-1} \cdot$ day $^{-1}$ prednisone, mycophenolic acid, tacrolimus, inhaled steroids, bronchodilators and low-dose macrolides. Despite this treatment, both her clinical condition and her PFT remained unchanged 3 months later: FEV1 $0.66 \mathrm{~L}$ (22\% pred) and FVC $1.63 \mathrm{~L}$ (46\% pred). At this time, she was given two infusions of rituximab (1,000 $\mathrm{mg}$ each) on days 1 and 15. Her pulmonary condition did not improve over the following months. The dyspnoea became increasingly disabling, and her PFT results progressively deteriorated (fig. 2). Hypercapnia eventually developed. The patient finally died of respiratory insufficiency 10 months after receiving rituximab.

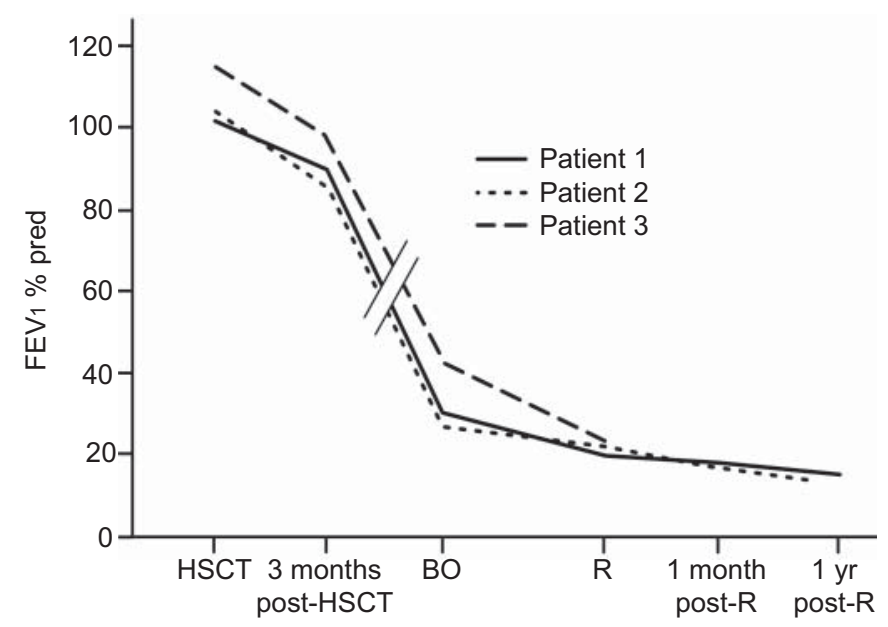

FIGURE 2. Evolution of patients' lung function testing. FEV1: forced expiratory volume $1 \mathrm{~s}$; \% pred: \% predicted; HSCT: haematopoietic stem cell transplantation; BO: bronchiolitis obliterans; R: rituximab.
Patient 3 was a 32-yr-old male who received PBSCs from an unrelated HLA-matched donor for Hodgkin's lymphoma after a reduced-intensity conditioning regimen that included total body irradiation and fludarabine. The course was complicated soon after transplantation by acute skin and ocular GVHD, which were improved through treatment with high-dose steroids. 3 months after transplantation, he developed a febrile pneumonia, which was resolved with empirical broad-spectrum antibiotics. 3 months later, while his skin GVHD was being controlled by $25 \mathrm{mg} \cdot$ day $^{-1}$ prednisone and $50 \mathrm{mg} \cdot$ day $^{-1}$ cyclosporin, he complained of dyspnoea and a productive cough. PFT revealed a new obstructive pattern with lung hyperinflation: FVC 4.98 L (89\% pred), FEV1 2.02 L (43\% pred), FEV1/FVC $40 \%$ and RV 3.32 L (175\% pred). A mosaic pattern of lung attenuation was observed on the lung CT scan. A lung infection was ruled out, and the diagnosis of BOS was maintained. Prednisone was increased to $1 \mathrm{mg} \cdot \mathrm{kg}^{-1} \cdot \mathrm{day}^{-1}$; in addition, inhaled steroids, bronchodilators and low-dose macrolides were added to the patient's cyclosporin regimen. Unfortunately, the patient's clinical course gradually worsened. 4 months later, while he was receiving $25 \mathrm{mg} \cdot \mathrm{day}^{-1}$ prednisone and cyclosporin, he had dyspnoea at rest, and his PFT revealed an FVC of $2.34 \mathrm{~L}$ (42\% pred) and an FEV1 of $1.04 \mathrm{~L}(22 \%$ pred). At this time, the patient had a recurrence of the skin and ocular GVHD. The patient was given three pulses of methylprednisolone (500 $\mathrm{mg}$ each) and no efficacy was observed by PFT 2 months later (fig. 2). He then received two infusions of rituximab (1,000 mg each) on days 1 and 15 . The patient died of respiratory distress 2 months later.

Since the demonstration of B-lymphocyte involvement in the pathophysiology of GVHD, few studies have assessed the efficacy of rituximab in steroid-refractory GVHD [4-6]. A recent review of these studies pointed out that the response to rituximab may depend on the affected organ, with the lung appearing to be among the less treatment-responsive organs [5]. However, these studies did not focus on lung evaluation, and the type of lung involvement was not specified for these patients or for others included in a recent prospective study $[5,6]$. Indeed, in some of these studies, one could postulate that lung involvement may have been a restrictive defect related to skin sclerodermoid GVHD [4], organising pneumonia or BO, as each of the patterns attributed to GVHD exhibit different characteristics and prognoses [1, 2,7]. Similarly, the criteria used to assess the lung's response to treatment in the mentioned studies were not clearly defined $[5,6]$.

However, the schedule used in patients with chronic GVHD (i.e. $375 \mathrm{mg} \cdot \mathrm{m}^{-2}$ weekly) might not be optimal for lung involvement. Because improvement of lung manifestations in patients with connective tissue diseases have been reported with rituximab at 1,000 mg on days 1 and 15 [8-10], we decided to use a similar schedule for our patients.

Herein, we report one case in which BO was histologically proven and two cases of patients who exhibited well-defined BOS. We also present precise data concerning follow-up PFT results after rituximab treatment. For the first time, we have demonstrated that the peribronchiolar inflammatory infiltrate of BO may be composed of several B-lymphocytes. Despite this feature, none of the patients responded to rituximab treatment. However, one limitation of our study is that all three patients 
received rituximab treatment in response to a severe obstructive ventilatory defect and, thus, our results do not preclude the potential efficacy of rituximab in less severe BO. Further studies are needed to determine whether rituximab is a valuable therapeutic option in chronic lung GVHD.

\section{G. Lorillon*, M. Robin", V. Meignin ", P. Ribaud", B. Lescoeur ${ }^{+}$, D. Gossot ${ }^{\S}$, G. Socié\#, A. Tazi* and A. Bergeron*}

*Service de Pneumologie, "Service d'Hématologie-Greffe, 'Service de Pathologie, Hôpital Saint-Louis, Université Paris 7, UFR Denis Diderot; Assistance Publique-Hôpitaux de Paris, +Service d'Hématologie Pédiatrique, Hôpital Robert Debré, Université Paris 7, UFR Denis Diderot; Assistance PubliqueHôpitaux de Paris, and ${ }^{\S}$ Service de Chirurgie Thoracique, Institut Mutualiste Montsouris, Paris, France.

Correspondence: A. Bergeron, Service de Pneumologie, Hôpital Saint-Louis, 1 avenue Claude Vellefaux, 75475, Paris cedex 10, France. E-mail: anne.bergeron-lafaurie@sls.aphp.fr

Statement of Interest: None declared.

\section{REFERENCES}

1 Filipovich AH, Weisdorf D, Pavletic S, et al. National Institutes of Health consensus development project on criteria for clinical trials in chronic graft-versus-host disease: I. Diagnosis and staging working group report. Biol Blood Marrow Transplant 2005; 11: 945-956.
2 Williams KM, Chien JW, Gladwin MT, et al. Bronchiolitis obliterans after allogeneic hematopoietic stem cell transplantation. JAMA 2009; 302: 306-314.

3 Dudek AZ, Mahaseth H, DeFor TE, et al. Bronchiolitis obliterans in chronic graft-versus-host disease: analysis of risk factors and treatment outcomes. Biol Blood Marrow Transplant 2003; 9: 657-666.

4 Ratanatharathorn V, Ayash L, Reynolds C, et al. Treatment of chronic graft-versus-host disease with anti-CD20 chimeric monoclonal antibody. Biol Blood Marrow Transplant 2003; 9: 505-511.

5 Kharfan-Dabaja MA, Mhaskar AR, Djulbegovic B, et al. Efficacy of rituximab in the setting of steroid-refractory chronic graft-versushost disease: a systematic review and meta-analysis. Biol Blood Marrow Transplant 2009; 15: 1005-1013.

6 Kim SJ, Lee JW, Jung CW, et al. Weekly rituximab followed by monthly rituximab treatment for steroid-refractory chronic graftversus-host disease: results from a prospective multicenter phase II study. Haematologica 2010; 95: 1935-1942.

7 Yoshihara S, Yanik G, Cooke KR, et al. Bronchiolitis obliterans syndrome (BOS), bronchiolitis obliterans organizing pneumonia (BOOP), and other late-onset noninfectious pulmonary complications following allogeneic hematopoietic stem cell transplantation. Biol Blood Marrow Transplant 2007; 13: 749-759.

8 McGonagle D, Tan AL, Madden J, et al. Successful treatment of resistant scleroderma-associated interstitial lung disease with rituximab. Rheumatology (Oxford) 2008; 47: 552-553.

9 Borie R, Debray MP, Laine C, et al. Rituximab therapy in autoimmune pulmonary alveolar proteinosis. Eur Respir J 2009; 33: 1503-1506.

10 Vandenbroucke E, Grutters JC, Altenburg J, et al. Rituximab in life threatening antisynthetase syndrome. Rheumatol Int 2009; 29: 1499-1502.

\section{Exclusive viral wheeze and allergic wheeze: evidence for discrete phenotypes}

\section{To the Editors:}

There is a broad consensus that childhood wheezing illness consists of several distinct disease entities, but there is no agreement on their number or underlying mechanisms [1]. Commonly used phenotypic classifications are based on clinical or epidemiological criteria [2-6], and there has been little work focusing on causal mechanisms $[1,6,7]$.

Trigger factors for wheezing episodes might be indicators of such mechanisms. In young children, wheeze is commonly triggered by respiratory viral infections (colds), physical factors associated with increased breathing (exercise, laughing, crying and excitement) or allergens (aeroallergens and food allergens). These triggers are increasingly used to define asthma phenotypes in young children [3,5]. Despite this, there are few data on the way in which these triggers change in importance with age or whether different classes of triggers are independent. A close association between different classes of triggers in the same children would suggest a common underlying mechanism while a lack of association might reflect independent causal mechanisms. In this analysis, we describe changes in the prevalence of different parent-reported triggers of wheeze by age in 1-9 yr olds and report statistical associations between different classes of triggers (exercise, allergens and infection).

We used data from the Leicestershire Cohort Studies, a population-based random sample of children living in Leicestershire, UK [8]. The children were recruited in 1998 at an age of 1-4 yrs and were followed up in 1999, 2001, 2003 and 2006. In each survey, their parents completed a postal questionnaire asking detailed questions about respiratory symptoms during the previous 12 months and environmental exposures. The Leicestershire Health Authority Research Ethics Committee (Leicester, UK) approved the study.

For ages 1, 4, 6 and 9 yrs, we assessed the prevalence of different classes of triggers of wheeze and their combinations among children reporting any wheeze in the previous 12 months. We considered the following classes of triggers reported by parents: exercise (running/playing and/or laughing/crying/excitement), 